\title{
Interruption Management: The Use of Attention-Directing Tactile Cues
}

\author{
Pamela J. Hopp, C. A. P. Smith, Benjamin A. Clegg, and Eric D. Heggestad, Colorado \\ State University, Fort Collins, Colorado
}

\begin{abstract}
Previous research has suggested that providing informative cues about interrupting stimuli aids management of multiple tasks. However, auditory and visual cues can be ineffective in certain situations. The objective of the present study was to explore whether attention-directing tactile cues aid or interfere with performance. A two-group posttest-only randomized experiment was conducted. Sixty-one participants completed a 30-min performance session consisting of aircraft-monitoring and gauge-reading computer tasks. Tactile signals were administered to a treatment group to indicate the arrival and location of interrupting tasks. Control participants had to remember to visually check for the interrupting tasks. Participants in the treatment group responded to more interrupting tasks and responded faster than did control participants. Groups did not differ on error rates for the interrupting tasks, performance of the primary task, or subjective workload perceptions. In the context of the tasks used in the present research, tactile cues allowed participants to effectively direct attention where needed without disrupting ongoing information processing. Tactile cues should be explored in a variety of other visual, interruptladen environments. Potential applications exist for aviation, user-interface design, vigilance tasks, and team environments.
\end{abstract}

\section{INTRODUCTION}

Task interruption in the workplace is a widespread phenomenon (e.g., see Mintzberg, 1973). Interruptions can have significant effects on both job performance and employee well-being. Perlow (1999), for example, found that software engineers in a high-tech firm had difficulty meeting deadlines because of the frequency of interruptions. In addition, Kirmeyer (1988) found that interruptions can increase perceived workload and job stress. More seriously, however, a failure to appropriately attend to interrupting stimuli can have life-threatening consequences in many domains (e.g., Dornheim, 2000). As a rather dramatic example, Griffon-Fouco and Ghertman (1984) found that job performance interruptions accounted for more than $15 \%$ of all shutdowns of nuclear power plants.

Interruptions occur in the context of ongoing task performance and can be classified into two broad categories (Fisher, 1998): externally generated and internally generated. Internally generated interruptions include things such as daydreams and intrusive thoughts, whereas externally generated interruptions include events as varied as a phone call, the arrival of an Email, a coworker with a question, and even a fire alarm. In this paper, we focus on a specific form of external interruption, in which the interruption comes from a secondary task within a multitask environment. In this context, the secondary task is one that does not require continuous attention but does need to be monitored and does require at least occasional action on the part of the operator. As such, an interruption occurs when the operator must divert attention from a continuous primary task to monitor and/or take action on a secondary task. The decision involved in whether and when to switch tasks differentiates interruptions from alarms, which typically require imperative attention (Sarter, 2001). 
Previous work has often framed the issue of task switching in terms of whether or not operators sample in an optimal fashion from a variety of channels of information (for reviews see Moray, 1981, 1986). Research on task-switching processes has shown that people struggle to remember to switch between operations unless there is a reminder in the environment indicating the need to do so (Spector \& Biederman, 1976). As such, performance on the switched task often suffers. One suggestion for improving performance on an interruptive secondary task has been to provide the individual with a cue signaling when the secondary task requires attention. It is reasoned that the inclusion of the cue allows the person to efficiently inhibit and activate the resources needed to process the tasks (Cellier \& Eyrolle, 1992). In the applied context of air traffic control, for instance, Ho, Nikolic, and Sarter (2001) and Ho, Waters, Nikolic, and Sarter (2003) demonstrated that performance was improved by providing cues indicating the nature and urgency of pending tasks.

The current study examines interruption management, the detection, interpretation, and integration of interruptions within ongoing task performance (Latorella, 1996). An ideal form of interruption is one that minimally distracts ongoing task performance while providing a clear signal of another source requiring the individual's attention (see Jett \& George, 2003, for a discussion of distraction). The optimal nature of a cue indicating that an interruptive secondary task needs attention remains an unresolved issue. Advantages and disadvantages of conveying information through various sensory modalities are found in research on sensory feedback (e.g., Sklar \& Sarter, 1999) and alarms (e.g., Sorkin, Kantowitz, \& Kantowitz, 1988). Because many tasks demand visual information processing, the use of visual cues may not be optimal; operators may suffer visual fatigue from an overdependence on the visual channel (Akamatsu, MacKenzie, \& Hasbroucq, 1995). The use of auditory cues has also been found to be limited. Specifically, some auditory cues are difficult to identify or recognize (Edworthy, Stanton, \& Hellier, 1995), whereas others are too intrusive and difficult to suppress (Sarter, 2000). Given the apparent limitations of visual and auditory cues, some attention has been given of late to provid- ing cues through the tactile modality. The purpose of the present study is to evaluate the effectiveness of tactile cues for signaling the arrival of an interruptive secondary task within the context of a complex multitask environment.

\section{Tactile Cues}

Researchers have begun to investigate the viability of using tactile cues in multiple-task environments. Several potential advantages of the tactile modality over the visual and auditory modalities have been identified: For example, the tactile channel is not heavily used, tactile cues can be readily detected, a limited vocabulary can be transmitted, the cues are not highly intrusive, tactile information can be omnidirectional, and tactile information can be perceived along with additional visual and auditory information (Gilliland \& Schlegel, 1994; Sarter, 2000; Sklar \& Sarter, 1999). Research on tactile communication indicates that it has potential to overcome some of the typical problems encountered by pilots and air traffic controllers (e.g., Zlotnik, 1988). For example, Sklar and Sarter found that flight instructors receiving tactile feedback were more accurate, quicker to react, and able to handle more concurrent workload demands than were those who received visual feedback.

However, research on the use of tactile cues has not shown universal benefits. Both Sklar and Sarter's (1999) and Zlotnik's (1988) research revealed some difficulties in the detection of tactile cues on the wrist and arm. Additionally, Gilliland and Schlegel (1994) found that tactile stimulation to the head degraded performance of concurrent tasks. One potential way to account for such findings is through Sanders's (1983) cognitive-energetical stage model. The model suggests that the arousing characteristics of tactile stimulation may interfere with other modes of communication or performance, particularly in more complex tasks. Specifically, Sanders pointed out that overstimulation of the arousal system can lead to immediate response action, resulting in rapid but careless responses. He also noted that tactile signals are like auditory signals in that they can be experienced as particularly arousing and that the effects of signal intensity and time uncertainty are additive. Therefore, it may be that a tactile cue under 
conditions of time uncertainty would be experienced as a particularly disruptive event and may lead to more errors.

\section{The Present Study and Hypotheses}

The present study addresses the following primary research question: Do directional tactile cues that prompt users to switch their attention provide performance benefits or create additional interference in an interrupt-laden environment? In particular, objective performance was evaluated on the serial processing of two distinct tasks. The first task was a continuous aircraftmonitoring task presented on a radar screen displayed on a computer monitor placed directly in front of the participant. A second task, interruptive gauge reading, was presented on computer monitors that were placed on either side of the participants, outside their field of peripheral vision. The control group was instructed to remember to check the side screens for potential interruptions, whereas the treatment group received directional tactile cues indicating the arrival and location of the interruptive task.

As discussed earlier, tactile cues may provide efficient reminders to perform interrupting tasks. Therefore, participants who receive tactile cues should demonstrate more efficient switching between tasks. Specifically, participants in the treatment group should attempt a greater proportion of interrupting tasks as compared with the control group. Additionally, the average time for participants to respond with a correct answer should be shorter in the treatment group.

Although tactile cues are predicted to alter the task-switching process, their presence is not expected to cause decrements in performance on either task. Typically, the introduction of another task causes performance of the ongoing task to suffer, resulting in a performance trade-off (Gopher, 1993). However, according to multiple resource theory (Wickens, 1984), tasks that require nonoverlapping resources should not interfere with each other. Thus the tactile cues should not interfere with the visual or psychomotor processing required by the two tasks. In particular, numbers of errors on the interruptive gauge task are expected to be equal in the treatment and control groups. Likewise, the numbers of hits, misses, false alarms, and correct rejections on the primary aircraft-monitoring task are expected to be the same in both groups. We recognize that such expectations about performance lead us to predict a null hypothesis. However, undisturbed performance is one indication of a successful interruption management strategy.

Participants were also asked to self-report perceptions of workload and task difficulty. Groups using different multitasking strategies have been found to perceive workload and task difficulty differently (Damos, 1984). It is therefore plausible that participants in this study would have different experiences of workload depending on the presence or absence of tactile aids. The presence of additional information in the form of tactile cues need not necessarily reduce workload. Wickens and Liu (1988) suggested a number of avenues by which the presentation of a simultaneous cross-modal stimulus, such as a tactile cue, might induce cognitive costs. However, based on previous evidence of noninterfering tactile feedback (e.g., Sklar \& Sarter, 1999), and because cued participants do not have the additional memory burden of remembering to switch tasks, participants receiving the tactile cues are expected to experience lower workload and stress than control participants, who must remember to monitor the side screens.

\section{METHODS}

\section{Participants}

The participants were 61 undergraduates enrolled in an introductory psychology course at a large, public university and who participated for partial course credit in a general psychology course. The sample consisted of 20 men, 39 women, and 2 students who did not identify their sex. Participants were randomly assigned to the treatment group $(n=29)$ or the control group $(n=32)$.

\section{Equipment}

The experiment was conducted on a threemonitor computer system equipped with software programmed in Visual BASIC. The system was set up so that the main task was presented on a center screen placed directly in front of the participant. Two additional monitors presented the interruptive task and were positioned 
laterally, one on each side of the participant, perpendicular to the center screen. The side screens were placed slightly to the rear of the participant's seat, outside his or her peripheral vision. As such, in order to observe the arrival of a gauge task question, participants were required to turn their head and scan the screens. The keyboard was placed in front of the participant and was used to respond to the interruptive task. The mouse was placed alongside the keyboard and was used to respond to the primary task.

The simple tactile stimuli were delivered through pager buzzers sewn into the shoulders of a vest. Cholewiak and Collins (2000) suggested that the torso is an effective presentation site that permits freedom of movement for the hands and arms. The buzzers were vibration motors of the type commonly used in cell phones and pagers. Buzzers were powered by a $1.5-\mathrm{V}$ D-cell battery; the characteristic frequency of vibration was approximately 30 cycles/s. The Visual BASIC program activated one of the buzzers at the appearance of the interrupting task. The buzzers vibrated for approximately $1 \mathrm{~s}$ on the side at which the task appeared.

\section{Task Paradigm}

The task required participants to engage in a continuous, visual, primary task modeled after an aircraft monitoring scenario. The interruptive task, consisting of a discrete, visual gaugereading task, appeared periodically throughout the scenario, interchanging pseudo-randomly between the left and right side screens. The task paradigm was pilot tested twice on groups of students to assess the difficulty level of scenarios. The scenarios were designed to be challenging enough that participants could not complete the task with complete accuracy and yet not be so difficult as to discourage participants from investing effort in the task.

Aircraft-monitoring task. Three scenarios were designed to be of equal difficulty. Each scenario was $10 \mathrm{~min}$ in length and presented 190 aircraft. The symbols used to represent aircraft were adapted from MIL-STD-2525 and consisted of an outer shape, a color, and an inner shape. For example, a symbol might be a square filled with a blue color and with a black dot in the center. Symbols were classified by the following characteristics: Any green symbol was "friendly," and any red symbol was "hostile," regardless of outer or inner shape. Any symbol having a dot as its inner shape was "perceived friendly," and any symbol having a cross as its inner shape was "perceived hostile," regardless of color or outer shape. Categorizations of symbols were exclusive such that no symbol fit more than one category (e.g., none featured a green symbol with a dot inside). Scenarios were designed with an equivalent number and rate of events so that the opportunities for action did not differ across scenarios.

The primary task screen, shown in Figure 1, consisted of a map with two circles. The outer circle was yellow and represented the warning zone. The inner circle was red and represented the firing zone. The warning button and fire buttons were displayed at the bottom of the screen. The participants were instructed to monitor the aircraft as they appeared on the screen and make decisions regarding the action needed based on symbol type. Friendly and perceived friendly aircraft were not to be acted upon. Hostile aircraft were to be fired upon if they entered the red circle. Perceived hostile aircraft were to be warned first if they entered the yellow circle and then fired upon if they entered the red circle. Participants were instructed to click on the symbol with the mouse in order to select it before any action button could be used. Once fired upon, the symbol displayed a graphic explosion and disappeared from the screen.

The aircraft-monitoring task consisted of visual input and required a considerable manual response load. Within each 60-s period, the number of planes that entered view ranged from 10 to 30 , with the number of those being hostile or potentially hostile ranging from 7 to 17 . On average, two thirds of the planes that crossed the screen required action from the participant. The planes took varying courses of action: Some flew in straight lines across the screen, some flew into the warning circle and left, some flew through the warning circle into the firing circle in varying patterns, and some flew around the periphery. A random sample of six trials was selected to provide an estimate of actions performed. Within this sample of six trials, participants averaged 31 mouse clicks/min, with variations ranging from 18 to 64 mouse clicks/min.

The computer tracked all actions taken by the 


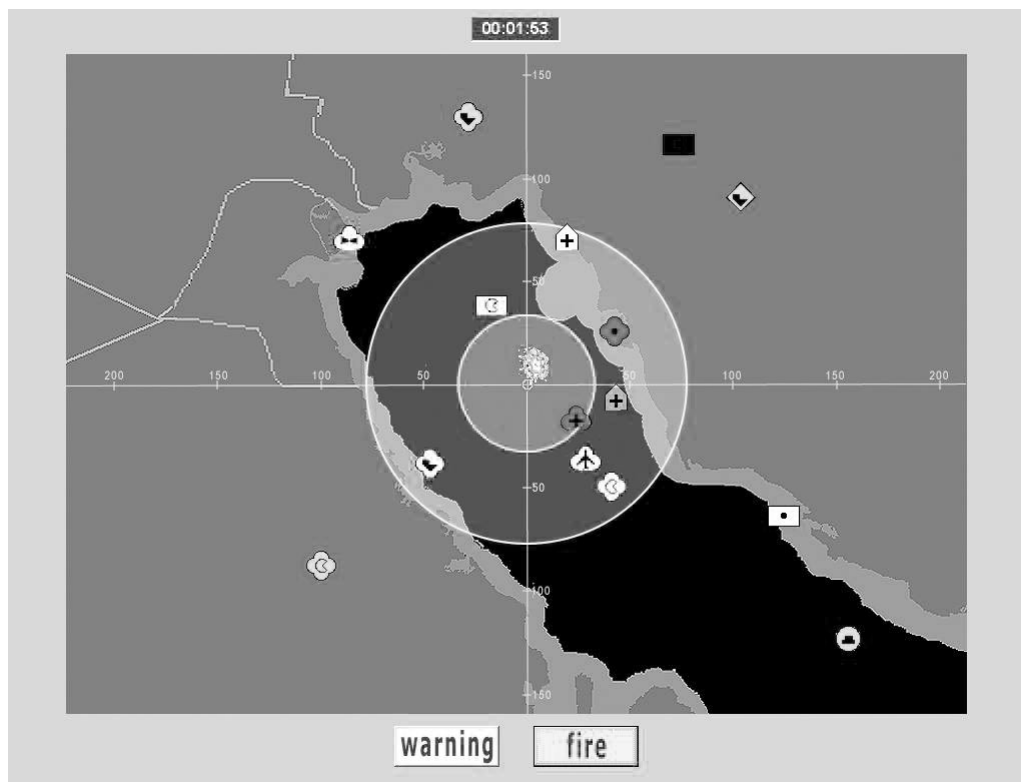

Figure 1. Example display from the primary task, aircraft monitoring.

participant and displayed a summarized count of correct decisions and errors made at the end of each scenario. For each participant, the program created a results file that tracked the dependent variables for this task, which were the number of hits, misses, false alarms, and correct rejections for each scenario. Hits occurred when participants correctly took action; misses occurred when participants failed to take action; false alarms occurred when participants erroneously took action; and correct rejections occurred when participants correctly restrained action.

Gauge-reading task. Fifteen gauge task interruptions were presented in each scenario. The gauge task consisted of a screen showing two gauges side by side (see Figure 2). Beneath the gauges, a question was presented that asked about the relative gauge readings. There were six possible question types that required a "yes" or "no" answer, indicated by pressing " $y$ " or " $n$ " on the keyboard. The questions consisted of any combination of whether the gauges were more or less than 5, 10, or 15 units apart. Question types and locations (left or right screen) were counterbalanced, with all permutations presented equally within each scenario. The time between questions ranged from 20 to $70 \mathrm{~s}$, and the duration that questions were displayed ranged between 10 and $20 \mathrm{~s}$. Hence the participant did not know which type of question would appear, when to anticipate a gauge question, to which side to expect its appearance, or for how long the question would remain visible.

The computer recorded the accuracy of each response as well as the response time in seconds. The number of questions answered correctly was displayed to the participant at the end of each scenario. The dependent variables used for this task were the proportion of questions attempted (number attempted divided by the total presented), the proportion of questions

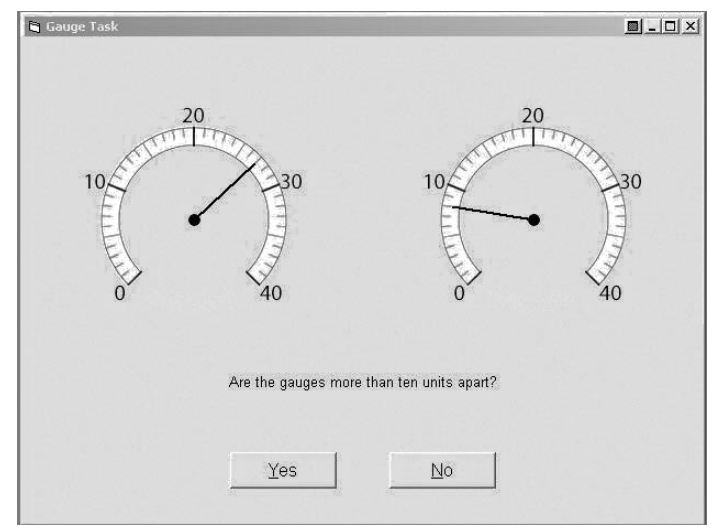

Figure 2. Example display from the interruptive task, gauge reading. 
answered incorrectly (number of incorrect responses divided by the number of questions attempted), and the average response time for correct responses (the seconds elapsed from appearance of the question to a correct response). The amount of time taken by participants to generate answers to the questions once they are aware of them should remain constant across groups; therefore any differences found in response times can be attributed to differences in attention-switching behavior.

\section{Experimental Conditions}

The control group consisted of 32 participants who were instructed to perform the aircraftmonitoring task while periodically checking the side screens for the appearance of the gauge task. The treatment group consisted of 29 participants who performed the same task but with the aid of tactile cues. The treatment group participants wore the vest equipped with pager buzzers. The treatment group was instructed to perform the aircraft-monitoring task and attend to the gauge task at the sensation of each buzz.

\section{Subjective Workload Measure}

A questionnaire was created to gather participant reactions to the task paradigm. Participants in the control group responded to eight questions using a 10-point Likert-type response scale. Items required participants to rate the difficulty of both tasks separately and in combination, time pressure, subjective performance, mental effort, frustration/stress, and physical discomfort. Participants in the treatment group responded to the same eight questions as well as an additional four questions inquiring about the tactile condition. The additional questions asked participants about the helpfulness and annoyance of the cues as well as whether they immediately switched their attention upon sensing the cue or waited for an opportune time to switch.

\section{Procedure}

Participants performed in a single session lasting approximately $50 \mathrm{~min}$. Participants were seated in front of the central monitor on a nonswiveling chair. They were then given an instruction sheet detailing the directions for both tasks.

The participants completed a 2-min training session on the aircraft-monitoring task. The software indicated if they made any mistakes on the training session, and the experimenter asked if they felt comfortable with the instructions. Participants then completed a 2-min training session on the gauge task. Again, the software indicated if they made any errors, and the experimenter confirmed their understanding of the instructions before proceeding. The experiment comprised three 10-min scenarios that included both the aircraft-monitoring and the gaugereading tasks. Scenario order was counterbalanced across participants. At the end of the first scenario, the scores for both tasks were displayed and participants were given a 1-min rest break. Participants then completed two more 10-min scenarios, with 1 -min rest breaks between trials. At the end of the third scenario, participants filled out the subjective workload questionnaire.

Performance throughout the entire 30-min situation was the criterion of interest. Therefore, performance measures were summed or averaged across all three trials. Within-subject variations of performance across trials were not of critical importance to the primary research question and for brevity will not be discussed.

\section{RESULTS}

\section{Gauge Task Performance}

The first question examined was whether or not the presence of tactile stimuli provided an effective cue to perform the gauge-reading task. Performance on the interruptive gauge task was analyzed using $t$ tests to compare the treatment group with the control group. Results indicated that the treatment group attempted a significantly greater proportion of gauge questions (.94 and .86 for the treatment and control groups, respectively), $t(45)=3.64, p<.05$, and responded significantly faster (5.92 and $6.74 \mathrm{~s}$ for the treatment and control groups, respectively), $t(59)=$ $-2.30, p<.05$, than did the control group. In contrast to these findings, no differences were found between the groups with respect to error rates (.17 and .19 for the treatment and control groups, respectively), $t(59)=-0.80, p>$ .05 . Taken together, these findings suggest that task switching was more effective in the treatment group and that the tactile cues did not interfere with the information-processing requirements of the interruptive task. 


\section{Monitoring Task Performance}

Because the previous analyses showed that tactile cues enhanced performance of the gauge task, the next issue was whether or not this interruption came at the expense of performance on the aircraft-monitoring task. In taking action against the hostile and perceived hostile objects, participants' actions were classified as hits, misses, false alarms, or correct rejections (see Table1). Hit rates were calculated by summing the total number of hits throughout the scenarios and dividing by the total number of times action was required. False alarm rates were calculated by summing the total number of false alarms throughout the scenario and dividing by the total number of times action was not required.

Performance on the aircraft-monitoring task was assessed using signal detection theory. Signal detection theory provides a method of assessing the decision-making process when evaluating different classes of items. This method provides a measure of the participants' sensitivity, or their ability to detect a signal, as well a measure of criterion, or bias in favoring a particular type of response. In the present task paradigm, the signal refers to the object type and accurate detection was shown by taking the appropriate action.

The hit rates and false alarm rates were used to calculate the sensitivity and criterion for each group. The sensitivity statistic represents the standardized difference between the means of the signal-present and signal-absent distributions.
Thus larger values indicate greater sensitivity in detecting a difference between distributions. Both groups were found to have a sensitivity measure of $d^{\prime}=2.35$. That the two groups had the same level of sensitivity indicates that both groups were equally able to identify and act upon the objects.

The criterion represents a bias toward either a Type I or Type II error - that is, a preference for false alarms or misses. A miss error represents allowing oneself to be attacked. A false alarm represents shooting at a nonhostile aircraft. In our study, neither the treatment nor the control groups exhibited an error bias $(\beta=$ 1.07 vs. 1.10 , respectively). The importance of this finding is that the presence of a tactile cue was not associated with a change in bias.

Finally a chi-square test examined whether the total number of hits, misses, false alarms, and correct rejections in the treatment and control groups differed from the expected values. The results, $\chi^{2}(3)=3.01, p>.05$, showed no significant difference in monitoring task performance. Therefore, no evidence was found to suggest a performance difference between groups on the aircraft-monitoring task.

Overall, these analyses suggest that the addition of the tactile cues did not adversely impact performance on the aircraft-monitoring task.

\section{Subjective Workload}

Participant responses on the subjective workload questionnaire (i.e., the eight questions

TABLE 1: Aircraft-Monitoring Task Performance

\begin{tabular}{|c|c|c|}
\hline \multirow{2}{*}{$\begin{array}{l}\text { Action } \\
\text { Taken }\end{array}$} & \multicolumn{2}{|c|}{ Action Required } \\
\hline & Yes & No \\
\hline \multicolumn{3}{|c|}{ Treatment Group $(n=29)$} \\
\hline Yes & $\begin{array}{l}\text { Hits: } 9431 \\
(89.4 \%)\end{array}$ & $\begin{array}{l}\text { False alarms: } 1018 \\
(13.7 \%)\end{array}$ \\
\hline No & $\begin{array}{l}\text { Misses: } 1113 \\
(10.6 \%)\end{array}$ & $\begin{array}{l}\text { Correct rejections: } 6436 \\
(86.3 \%)\end{array}$ \\
\hline \multicolumn{3}{|c|}{ Control Group ( $n=32$ ) } \\
\hline Yes & $\begin{array}{l}\text { Hits: } 10563 \\
(90.0 \%)\end{array}$ & $\begin{array}{l}\text { False alarms: } 1177 \\
(14.2 \%)\end{array}$ \\
\hline No & $\begin{array}{l}\text { Misses: } 1172 \\
(10.0 \%)\end{array}$ & $\begin{array}{l}\text { Correct rejections: } 7099 \\
(85.8 \%)\end{array}$ \\
\hline
\end{tabular}


in common across the two conditions) were summed to form a composite workload rating. No significant effect of group on subjective workload perceptions was observed (mean treatment $=$ 43.4 , mean control $=42.8), t(58)=0.25, p>.05$. The standardized mean difference effect size between the two groups on this measure was 0.07 standard deviations. As such, these data suggest that the tactile cues did not create substantial additional workload or less favorable conditions as compared with the control group.

Responses to the four treatment group questions regarding the tactile cues were also examined. The responses suggested that the tactile cues were helpful $(M=9.15$ on a 10-point scale, $S D=1.85, n=27)$ and of low annoyance $(M=$ $2.29, S D=2.46, n=28$ ). The majority of these participants $(71 \%)$ indicated that most of the time they waited to switch their attention until a desired action was completed. Thus the cues prepared people to switch attention but did apparently allow for some individual control in determining the optimal time to switch.

\section{DISCUSSION}

The central question within this study concerned the effectiveness of using tactile cues to manage the interruption of performance on an ongoing task in order to switch to a secondary activity. The results showed that using such cues led to more frequent and faster responding in the secondary task without any significant associated decrement in the central task. In addition, self-reports of those who received tactile cues indicated that they did not perceive the multipletask environment as more difficult than did those who did not receive tactile cues. These findings suggest that tactile cues offer a viable mechanism to signal the need to direct attention to a secondary task in the context of multiple-task performance.

\section{Impact of Tactile Cues on Gauge Task Performance}

The present results provide evidence in support of the use of tactile cues in a multiple-task environment, but they do not directly resolve the psychological processes through which the cues aided performance of the secondary task. We speculate, however, that cues may have re- sulted in a change in the nature of the secondary task. Memory researchers studying prospective memory, or the need to perform an intended action in the future, have distinguished between two types of tasks: time-based and event-based prospective memory tasks. Time-based tasks are tasks in which the elapse of a certain period determines the need to perform the intended action. Conversely, event-based tasks are signaled by external events and thereby provide greater environmental support than do timebased tasks (Kliegel, Martin, McDaniel, \& Einstein, 2002). In this study, it is possible that the tactile cues transformed the task switching from a time-based task, in which participants had to remember to periodically check the side screens, into an event-based task, in which participants relied on the cues to signal the need to shift attention. McDaniel and Einstein (2000) discussed how, within event-based tasks, it is possible to use relatively resource-free processes to support prospective memory. Therefore tactile cuing may be a resource-efficient method of structuring attention in a busy visual environment.

Although providing tactile cues enhanced performance on the secondary task, participants did not respond to all of the gauge question occurrences. Thus, although the presence of the tactile cue improved the response rate to the gauge task, it did not lead to perfect performance. Failure to respond to a gauge task question among treatment group members may have been attributable to (a) failure to recognize the tactile cue or (b) a choice not to switch (presumably because of the demands of the aircraftmonitoring task at the time of the cue). The choice not to switch is consistent with work by McFarlane (2002), who examined a variety of methods of interruption. One method he examined was what he termed the negotiated interruption, a scenario in which the user can determine when to switch attention between the tasks and, as such, is comparable to the interruption employed in the present study. McFarlane found that negotiated interrupts resulted in poorer performance in regard to completeness and promptness of the interrupting tasks; the response rates were less than perfect. Thus McFarlane concluded that the negotiated method might result in people not handling interruptions in a timely manner. 


\section{Impact of Tactile Cues on Aircraft- Monitoring Task Performance}

An attention-switching cue is effective if it does not disrupt primary task performance. A major concern for tactile cues was whether they would interfere with primary task performance by being particularly disruptive (Sanders, 1983). Thus it was important that our data suggested that the tactile cues did not adversely affect primary task performance and that selfreported difficulty of the task environment did not differ between the two groups. In particular, the presence of tactile cues affected neither the participants' ability to identify threats nor their preference for one type of error over the other. Error preference, or bias, is especially important. For example, military decision makers often exhibit a very strong preference for miss errors, which is a rational response to the base-rate incidence of hostile and friendly aircraft in a busy airspace (Smith, Johnston, \& Paris, 2004). In this context, any decision aid that affects error preference would essentially alter an institutional policy - not a desirable feature for a decision aid. In the current study, the use of tactile cues was not associated with a change in error bias, which leads us to believe that tactile cuing is a promising technology for many high-stakes task domains.

The lack of interference created by tactile cues suggests that tactile communication might be advantageous in certain environments. However, Wickens's (2002) latest conceptualization of multiple resource theory does not include a set of resources allocated to the tactile modality. Perhaps a tactile component of information processing deserves specification in the model.

\section{Limitations}

Some cautionary notes are in order here, however. We do not know the extent to which these findings would generalize to different multiple-task environments. That is, it is possible that the use of tactile cues to prompt task switching may degrade primary task performance for different task combinations. Additionally, because we did not compare tactile cues with other types of cues, we cannot declare the superiority of tactile cues over visual or auditory cues.

\section{Future Directions}

One potential extension of this work is to explore the use of tactile cues conveying additional dimensions of information. For example, Ho et al. (2001, 2003) showed the benefits of providing informative cues regarding the nature of pending tasks. Tactile directional cues could be refined to also incorporate information about the nature and urgency of a task. Such cues could vary in frequency or intensity to distinguish among the importance of various interruptions. Ongoing research is examining the type of information that can be effectively transmitted via a tactile cue (e.g., see Rupert, 2000; van Erp, 2001) and the nature of tactile localization depending on the specific body placement (e.g., Cholewiak, Brill, \& Schwab, 2004; Cholewiak \& Collins, 2003).

Also, research might examine the benefits of tactile cues for more experienced operators. The current study was conducted on college students participating for course credit, using a simulated task. As discussed previously, research on task interference differs widely according to the specific nature of the tasks employed. Future work therefore is needed to examine the generalizability of the current findings to other task environments and to other types of users.

Finally, future work should examine other possible applications for tactile cues. Tactile interruption management should be explored in conjunction with user-interface development research. An intelligent attention-managing system could be designed to select the optimal modality for interrupt signals based on the nature of the tasks being performed. Tactile cues might also aid vigilance tasks to enhance detection and responsiveness to low base-rate events. Another option deserving exploration is the use of tactile cues in a team environment, in which it might be advantageous to have an unobtrusive method of communicating to certain team members without disrupting the entire team (Mark, 2002).

\section{Conclusion}

This study was unique in that it examined tactile priming of attention in a multiple-task environment. As Woods, Johannesen, Cook, and Sarter (1994) pointed out, attention control in 
the face of multiple tasks that compete for limited resources is the "least explored frontier in cognitive science and human-machine cooperation" (p. 67). Demands on attention will only increase in the future as technology increases the complexity of work environments. This study demonstrates that tactile cues may prove useful in directing attention to a noncontinuous secondary task when that task requires attention from the operator.

\section{ACKNOWLEDGMENTS}

This research was supported by the Defense Advanced Research Projects Agency Grant BAA01-038, Commander Dylan Schmorrow, Ph.D., U.S. Navy.

\section{REFERENCES}

Akamatsu, M., MacKenzie, I. S., \& Hasbroucq, T. (1995). A comparison of tactile, auditory, and visual feedback in a pointing task using a mouse-type device. Ergonomics, 38, 816-827.

Cellier, J.-M., \& Eyrolle, H. (1992). Interference between switched tasks. Ergonomics, 35, 25-36.

Cholewiak, R. W., Brill, J. C., \& Schwab, A. (2004). Vibrotactile localization on the abdomen: Effects of place and space. Perception and Psychophysics, 66, 970-987.

Cholewiak, R. W., \& Collins, A. A. (2000). The generation of vibrotactile patterns on a linear array: Influences of body site, time, and presentation mode. Perception and Psychophysics, $62,1220-1235$.

Cholewiak, R. W., \& Collins, A. A. (2003). Vibrotactile localization on the arm: Effects of place, space, and age. Perception and Psychophysics, 65, 1058-1077.

Damos, D. L. (1984). Individual differences in multiple-task performance and subjective estimates of workload. Perceptual and Motor Skill, 59, 567-580.

Dornheim, M. A. (2000, July 17). Crew distractions emerge as new safety focus. Aviation Week and Space Technology, pp. 58-60.

Edworthy, J., Stanton, N., \& Hellier, E. (1995). Warnings in research and practice [Editorial]. Ergonomics, 38, 2145-2154.

Fisher, C. D. (1998). Effects of external and internal interruptions on boredom at work: Two studies. Journal of Organizational Behavior, 19, 503-522.

Gilliland, K., \& Schlegel, R. E. (1994). Tactile stimulation of the human head for information display. Human Factors, 36, 700-717.

Gopher, D. (1993). The skill of attention control: Acquisition and execution of attention strategies. In D. E. Meyer \& S. Kornblum (Eds.), Attention and performance XIV: Synergies in experimental psychology, artificial intelligence, and cognitive neuroscience (pp. 299-322). Cambridge, MA: MIT Press.

Griffon-Fouco, M., \& Ghertman, F. (1984). Recueil de données sur les facteurs humains à Electricité de France [Collection of data on the human factors with Electricity of France]. In Operational safety of nuclear power plants (pp. 157-172). Vienna, Austria: Vienna International Atomic Energy Agency.

Ho, C.-Y., Nikolic, M. I., \& Sarter, N. B. (2001). Multimodal information presentation in support of timesharing and effective interruption management. In Proceedings of the 20th Digital Avionics Systems Conference (pp. 5D2/1-5D2/8). Los Alamitos, CA: Institute of Electrical \& Electronics Engineers.

Ho, C.-Y., Waters, M. J., Nikolic, M. I., \& Sarter, N. B. (2003, April). Supporting attention management in complex eventdriven domains through informative interruptive cueing. Paper presented at the 12th International Symposium on Aviation Psychology, Dayton, $\mathrm{OH}$.

Jett, Q. R., \& George, J. M. (2003). Work interrupted: A closer look at the role of interruptions in organizational life [Note] Academy of Management Review, 28, 494-507.

Kirmeyer, S. L. (1988). Coping with competing demands: Interruptions and the Type A pattern. Journal of Applied Psychology, 73, 621-629.

Kliegel, M., Martin, M., McDaniel, M. A., \& Einstein, G. O. (2002). Complex prospective memory and executive control of working memory: A process model. Psychologische Beitrage, 44, 303-318.

Latorella, K. A. (1996). Investigating interruptions: Implications for flight deck performance. Unpublished doctoral dissertation, State University of New York at Buffalo.

Mark, G. (2002). Extreme collaboration. Communications of the $A C M, 45(4), 89-93$.

McDaniel, M. A., \& Einstein, G. O. (2000). Strategic and automatic processes in prospective memory retrieval: A multiprocess framework. Applied Cognitive Psychology, 14, S127-S144.

McFarlane, D. C. (2002). Comparison of four primary methods for coordinating the interruption of people in human-computer interaction. Human-Computer Interaction, 17, 63-139.

Mintzberg, H. (1973). The nature of managerial work. New York: Harper and Row.

Moray, N. (1981). The role of attention in the detection of errors and the diagnosis of errors in man-machine systems. In J. Rasmussen \& W. Rouse (Eds.), Human detection and diagnosis of system failures (pp. 185-198). New York: Plenum.

Moray, N. (1986). Monitoring behavior and supervisory control. In K. R. Boff, L. Kaufman, \& J. P. Thomas (Eds.), Handbook of perception and human performance (pp. 40.1-40.51). New York: Wiley.

Perlow, L. (1999). The time famine: Toward a sociology of work time. Administrative Science Quarterly, 44, 57-81.

Rupert, A. H. (2000). An instrumentation solution for reducing spatial disorientation mishaps. IEEE Engineering in Medicine and Biology, 19(2), 71-80.

Sanders, A. F. (1983). Towards a model of stress and human performance. Acta Psychologica, 53, 61-97.

Sarter, N. B. (2000). The need for multisensory interfaces in support of effective attention allocation in highly dynamic eventdriven domains: The case of cockpit automation. International Journal of Aviation Psychology, 10, 231-245.

Sarter, N. B. (2001). Human technology interface: Multimodal communication in support of coordinative functions in humanmachine teams. Human Performance in Extreme Environments, 5(2), 50-54.

Sklar, A. E., \& Sarter, N. B. (1999). Good vibrations: Tactile feedback in support of attention allocation and human-automation coordination in event-driven domains. Human Factors, 41, 543-552.

Smith, C. A. P., Johnston, J., \& Paris, C. (2004). Decision support for air warfare: Detection of deceptive threats. Group Decision and Negotiation, 13, 129-148.

Sorkin, R. D., Kantowitz, B. H., \& Kantowitz, S. C. (1988). Likelihood alarm displays. Human Factors, 30, 445-459.

Spector, A., \& Biederman, I. (1976). Mental set and mental shift revisited. American Journal of Psychology, 89, 669-679.

van Erp, J. B. F. (2001). Tactile navigation display. In S. Brewster \& R. Murray-Smith (Eds.), Haptic human-computer interaction (pp. 165-173). Berlin: Springer Verlag.

Wickens, C. D. (1984). Processing resources in attention. In R. Parasuraman \& R. Davies (Eds.), Varieties of attention (pp. 63-101). Orlando, FL: Academic.

Wickens, C. D. (2002). Multiple resources and performance prediction. Theoretical Issues in Ergonomic Science, 3, 159-177.

Wickens, C. D., \& Liu, Y. (1988). Codes and modalities in multiple resources: A success and a qualification. Human Factors, 30, 599-616.

Woods, D. D., Johannesen, L., Cook, R. I., \& Sarter, N. (1994). Behind human error, cognitive systems, computers, and hindsight. Dayton, OH: Crew Systems Ergonomic Information and Analysis Center. 
Zlotnik, M. A. (1988). Applying electro-tactile display technology to fighter aircraft - Flying with feeling again. In Proceedings of the IEEE 1988 National Aerospace and Electronics Conference, NAECON 1988 (pp. 191-197). New York: IEEE Aerospace and Electronics Systems Society.

Pamela J. Hopp is a doctoral candidate in the industrial/organizational psychology program at Colorado State University, where she received her M.S. in psychology in 2003.

C. A. P. Smith is an assistant professor of computer information systems at Colorado State University. He received his Ph.D. in management information systems in 1990 from the University of Arizona.
Benjamin A. Clegg is an assistant professor of cognitive psychology at Colorado State University. He received his Ph.D. in psychology in 1998 from the University of Oregon.

Eric D. Heggestad is an assistant professor of industrial/organizational psychology at Colorado State University. He received his Ph.D. in psychology in 1997 from the University of Minnesota.

Date received: September 5, 2003

Date accepted: May 27, 2004 